\title{
Unrestricted treatment for the direct variational determination of the two- electron reduced density matrix for doubly occupied-configuration-interaction wave functions
}

Cite as: J. Chem. Phys. 150, 164106 (2019); https://doi.org/10.1063/1.5092182

Submitted: 08 February 2019. Accepted: 03 April 2019. Published Online: 23 April 2019

Diego R. Alcoba (D), Alicia Torre (D), Luis Lain (D), Gustavo E. Massaccesi, Ofelia B. Oña, and Elías Ríos

\section{ARTICLES YOU MAY BE INTERESTED IN}

Variational reduced density matrix method in the doubly occupied configuration interaction space using three-particle $\mathrm{N}$-representability conditions

The Journal of Chemical Physics 149, 194105 (2018); https://doi.org/10.1063/1.5056247

Range-separated multideterminant density-functional theory with a short-range correlation functional of the on-top pair density

The Journal of Chemical Physics 150, 084103 (2019); https://doi.org/10.1063/1.5082638

Sparse non-orthogonal wave function expansions from the extension of the generalized Pauli constraints to the two-electron reduced density matrix

The Journal of Chemical Physics 150, 144102 (2019); https://doi.org/10.1063/1.5085056

The Journal of Chemical Physics 


\title{
Unrestricted treatment for the direct variational determination of the two-electron reduced density matrix for doubly occupied-configuration-interaction wave functions
}

\author{
Cite as: J. Chem. Phys. 150, 164106 (2019); doi: 10.1063/1.5092182 \\ Submitted: 8 February 2019 • Accepted: 3 April 2019 • \\ Published Online: 22 April 2019
}

Diego R. Alcoba, ${ }^{1, a)}$ (D) Alicia Torre, ${ }^{2}$ (D) Luis Lain, ${ }^{2}$ (D) Gustavo E. Massaccesi, ${ }^{3}$ Ofelia B. Oña, ${ }^{4}$ and Elías Ríos ${ }^{4}$

\begin{abstract}
AFFILIATIONS
${ }^{1}$ Departamento de Física, Facultad de Ciencias Exactas y Naturales, Universidad de Buenos Aires and Instituto de Física de Buenos Aires, Consejo Nacional de Investigaciones Científicas y Técnicas. Ciudad Universitaria, 1428 Buenos Aires, Argentina

${ }^{2}$ Departamento de Química Física, Facultad de Ciencia y Tecnología, Universidad del País Vasco. Apdo. 644, E-48080 Bilbao, Spain

${ }^{3}$ Departamento de Ciencias Exactas, Ciclo Básico Común, Universidad de Buenos Aires. Ciudad Universitaria, 1428 Buenos Aires, Argentina

${ }^{4}$ Instituto de Investigaciones Fisicoquímicas Teóricas y Aplicadas, Universidad Nacional de La Plata, CCT La Plata, Consejo Nacional de Investigaciones Científicas y Técnicas. Diag. 113 y 64 (S/N), Sucursal 4, CC 16, 1900 La Plata, Argentina
\end{abstract}

a) Author to whom correspondence should be addressed: dalcoba@df.uba.ar

\begin{abstract}
This work extends to the unrestricted orbital approach the procedure described in our previous report [Alcoba et al., J. Chem. Phys. 148, 024105 (2018)] for determining variationally the two-electron reduced density matrix arising from doubly occupied-configuration-interaction wave functions by imposing two- and three-index $N$-representability conditions. An analysis of the numerical results obtained in selected systems, from both restricted and unrestricted treatments, allows one to assess the performance of these methodologies as well as to show the influence of the $P, Q, G, T 1$, and $T 2$ positivity conditions. We highlight the satisfactory results obtained within the unrestricted scheme.
\end{abstract}

Published under license by AIP Publishing. https://doi.org/10.1063/1.5092182

\section{INTRODUCTION}

Most Hamiltonians describing $N$-electron systems of chemical interest, such as atoms, molecules, and aggregates, are formulated in terms of two-particle interactions. Consequently, the two-electron reduced density matrix (2-RDM) constitutes a tool which contains all relevant information required to evaluate exactly all the fundamental electronic properties corresponding to the state of a system, including its energy. ${ }^{1}$ Moreover, the 2-RDMs avoid the explicit use of $N$-electron wave functions, for which the full configurationinteraction (FCI) determinantal expansion provides the exact formulation. In this context, the two-electron-reduced-density -matrix-based variational (v2RDM) method is the most intuitive treatment for determining ground-state energies of $\mathrm{N}$-electron systems; it consists in finding the 2-RDM elements which minimize the energy of a state. However, the attempts to determine energies following this procedure may lead to unphysical results. The reason for this failure is that the variational treatment itself does not guarantee that the obtained 2-RDM arises from an $\mathrm{N}$-electron density matrix (or from an $N$-electron wave function). This is known as the $N$-representability problem of the reduced density matrices. ${ }^{2,3}$ This drawback has been partially overcome by imposing constraints in the variational determination of the 2-RDM so that this matrix and other related ones turn out to be positive semidefinite. The so-called $P, Q$, and $G$ two-index $N$-representability conditions ${ }^{4}$ have been extensively used, and more recently, the $T 1$ and $T 2$ 
three-index ones ${ }^{5-9}$ have also been incorporated to these variational treatments.

From a computational point of view, the variational determination of the 2-RDM very often becomes impracticable due to its high cost, what raises the search for affordable approximations. For closed-shell systems, one alternative, in terms of $N$-electron wave functions, is to limit the configuration-interaction (CI) determinantal expansion to doubly occupied Slater determinants. This type of approximation for wave functions is known as doubly occupiedconfiguration-interaction (DOCI) expansion. ${ }^{10,11}$ The DOCI expansions correspond to Hilbert subspaces of seniority number zero, where the seniority number has been defined as the number of single occupied orbitals in a determinant, ${ }^{12-14}$ and constitute the first rung of the seniority-number-based CI treatments whose limit is the exact FCI solution. It has been proven that the DOCI wave functions capture most of the static correlation ${ }^{15-20}$ and are an alternative to excitation-based CI treatments in which the selection of determinants is performed according to their excitation level with respect to a reference determinant. ${ }^{21}$ The $N$-electron DOCI wave functions provide simpler structures of the 2-RDM and reduce the computational scaling of the variational method while retaining most of the static correlation. In previous studies, we have studied the variational determination of the 2-RDM elements corresponding to DOCI wave functions (v2RDM-DOCI) ${ }^{22-25}$ by imposing the above mentioned $N$-representability conditions by means of semidefinite programing algorithms (SDPAs) in which the energy, being a linear function of the $2-\mathrm{RDM}$, is minimized over the intersection of a linear affine space and the convex cone of block-diagonal positive semidefinite matrices. ${ }^{9,26}$ The DOCI procedure is basisset dependent since any unitary transformation of its orbital sets changes the energy of the $N$-electron system. Consequently, the implementation of this method requires the determination of orbital sets minimizing the energy ${ }^{15,27,28}$ or other quantities. ${ }^{16,29-31}$ Within the v2RDM-DOCI approach, this task has been carried out in the restricted orbital framework, in which the $\alpha$ - and $\beta$-spin-orbitals have identical spatial orbital component. ${ }^{25}$ The main aim of this work is to extend the v2RDM-DOCI methodology to a more general unrestricted treatment, where different orbitals breaking the spin symmetry are used for the $\alpha$ - and $\beta$-electrons, in order to know whether an independent determination of the $\alpha$ - and $\beta$-orbitals can achieve an energy lowering, approaching to the exact FCI value.

The organization of this work is as follows. In Sec. II, we report the main theoretical aspects used in this work as well as their mathematical formulations. Section III describes the computational details and the results obtained in several $\mathrm{H}_{4}$ clusters with different geometric arrangements, providing different degrees of orbital quasidegeneracy; we have chosen these challenging clusters, widely used in the literature, ${ }^{32-37}$ as test examples to check our proposals. The discussion of the results and their comparison with those obtained from the restricted treatment are also reported in this section. Finally, in Sec. IV, we highlight the main conclusions of this work.

\section{THEORETICAL FRAMEWORK}

In this work, we will describe quantum-mechanical operators in a space defined by a one-electron basis set $\left\{i_{1}^{\alpha}, i_{2}^{\alpha} \ldots i_{K}^{\alpha}, i_{1}^{\beta}, i_{2}^{\beta} \ldots i_{K}^{\beta}\right\}$, constituted by the union of two orthonormal basis subsets of $K \alpha$ spin orbitals $\left\{i_{1}^{\alpha}, i_{2}^{\alpha} \ldots i_{K}^{\alpha}\right\}$ and $K \beta$-spin orbitals $\left\{i_{1}^{\beta}, i_{2}^{\beta} \ldots i_{K}^{\beta}\right\}$; the orbital functions of the corresponding spin-orbitals $i_{j}^{\alpha}$ and $i_{j}^{\beta}$ are not necessarily identical nor orthogonal and similarly for the case $i_{j}^{\alpha}$ and $i_{k}^{\beta}(j \neq k)$.

Using the standard creation and annihilation fermion operators, $a_{i^{\sigma}}^{\dagger}$ and $a_{i^{\sigma}}$, respectively, ${ }^{38}$ the elements of the $q$-RDM, corresponding to an $N$-electron wave function $\Psi$, are defined as

$$
{ }^{q} D_{j_{1}^{\sigma_{1}} \ldots j_{q}^{\sigma_{q}}}^{i_{\sigma_{1}}^{\sigma_{1}} \ldots i_{q}^{\sigma_{q}}}=\left\langle\Psi\left|\frac{a_{i_{1}^{\sigma_{1}}}^{\dagger} \ldots a_{i_{q}}^{\dagger} a_{j_{q}}^{\dagger} a_{j_{q}} \ldots a_{j_{1}^{\sigma_{1}}}}{q !}\right| \Psi\right\rangle,
$$

where $\sigma_{1}, \ldots, \sigma_{q}$ are spin coordinates $(\alpha$ or $\beta$ ).

With that basis set and by means of those fermion operators, one can define an $N$-electron pseudo- $S_{z}$ operator ${ }^{p} \hat{S}_{z}$ as

$$
{ }^{p} \hat{S}_{z}=\frac{1}{2} \sum_{i}\left(a_{i^{\alpha}}^{\dagger} a_{i^{\alpha}}-a_{i^{\beta}}^{\dagger} a_{i^{\beta}}\right)
$$

Obviously, the ${ }^{p} \hat{S}_{z}$ operator coincides with the conventional- $S_{z}$ one $\hat{S}_{z}$.

Similarly, we can define the pseudo-spin-ladder operators

$$
{ }^{p} \hat{S}^{+}=\sum_{i} a_{i^{\alpha}}^{\dagger} a_{i \beta}
$$

and

$$
{ }^{p} \hat{S}^{-}=\sum_{i} a_{i \beta}^{\dagger} a_{i^{\alpha}},
$$

which allow one to formulate a pseudo-spin-squared operator as

$$
{ }^{p} \hat{S}^{2}={ }^{p} \hat{S}^{+p} \hat{S}^{-}+{ }^{p} \hat{S}_{z}^{2}-{ }^{p} \hat{S}_{z}={ }^{p} \hat{S}^{-p} \hat{S}^{+}+{ }^{p} \hat{S}_{z}^{2}+{ }^{p} \hat{S}_{z}
$$

The operators $\hat{S}^{2}$ and ${ }^{p} \hat{S}^{2}$ coincide when the spatial part of the spinorbitals $i_{j}^{\alpha}$ and $i_{j}^{\beta}$ is identical $(\forall j)$.

We will also define an $N$-electron pseudo-seniority-number operator $^{p} \hat{\Omega}$ by means of the basis set $\left\{i_{1}^{\alpha}, i_{2}^{\alpha} \ldots i_{K}^{\alpha}, i_{1}^{\beta}, i_{2}^{\beta} \ldots i_{K}^{\beta}\right\}$ as

$$
{ }^{p} \hat{\Omega}=\hat{N}-2 \sum_{i} a_{i^{\alpha}}^{\dagger} a_{i^{\beta}}^{\dagger} a_{i^{\beta}} a_{i^{\alpha}}
$$

where $\hat{N}$ stands for the electron number operator. This definition implies a kind of "pairing" between the spin orbitals $i_{j}^{\alpha}$ and $i_{j}^{\beta}, \forall j$, according to the values of the subscripts $j$ in their spinorbital basis subsets. Again, when the orbital functions of the spinorbitals $i_{j}^{\alpha}$ and $i_{j}^{\beta}$ are identical $(\forall j)$, the ${ }^{p} \hat{\Omega}$ operator coincides with the conventional-seniority-number one $\hat{\Omega} ;{ }^{16}$ the calculation of the expectation value of the $\hat{\Omega}$ operator is one of the procedures to determine the number of effectively unpaired electrons corresponding to an $N$-electron state. 39,40

Using the well-known commutation and anticommutation fermion operator rules, one can show that the following commutation relationships between operators are satisfied: 


$$
\begin{aligned}
& {\left[{ }^{p} \hat{S}_{z},{ }^{p} \hat{S}^{2}\right]=0,} \\
& {\left[{ }^{p} \hat{S}_{z},{ }^{p} \hat{\Omega}\right]=0,} \\
& {\left[{ }^{p} \hat{S}^{2},{ }^{p} \hat{\Omega}\right]=0 .}
\end{aligned}
$$

Hence, the commutation relationships between the operators ${ }^{p} \hat{S}_{z}$, ${ }^{P} \hat{S}^{2}$, and ${ }^{P} \hat{\Omega}$ are counterparts to the corresponding conventional ones, showing that the subspaces corresponding to the eigenvalues of these operators satisfy the relations reported in Refs. 17 and 19. In particular, an eigenfunction of the ${ }^{p} \hat{\Omega}$ operator of zero eigenvalue possesses identical eigenvalues for the ${ }^{p} \hat{S}_{z}$ and ${ }^{p} \hat{S}^{2}$ operators. It must be emphasized that a $\left\langle{ }^{p} \hat{\Omega}\right\rangle=0$ value implies a value $\left\langle{ }^{p} \hat{S}^{2}\right\rangle$ $=0$ but it does not necessarily imply a zero value for the $\left\langle\hat{S}^{2}\right\rangle$ quantity, what entails the appearance of spin contamination which could be reduced applying procedures dealing with wave functions ${ }^{41}$ or reduced density matrices. ${ }^{42-44}$ The $\left\langle\hat{S}^{2}\right\rangle$ values can be evaluated by means of the well-known relationship ${ }^{45,46}$

$$
\left\langle\hat{S}^{2}\right\rangle=\frac{N}{2}+\frac{\left(N_{\alpha}-N_{\beta}\right)^{2}}{4}+2 \sum_{i^{\alpha} j^{\alpha} k^{\beta} l^{\beta}} S_{l \beta}^{i^{\alpha}} S_{j^{\alpha}}^{k^{\beta}} D_{j^{\alpha} \beta^{\beta}}^{i^{\alpha} \alpha^{\beta}},
$$

in which $N_{\alpha}$ and $N_{\beta}$ are the number of $\alpha$ - and $\beta$-electrons of the system, respectively, $S_{l^{\beta}}^{\alpha^{\alpha}}$ and $S_{j^{\alpha}}^{k^{\beta}}$ are the overlap integrals of the spatial parts of the corresponding spin-orbitals, and ${ }^{2} D_{j^{\alpha} \alpha^{\beta}}^{i^{\alpha} k^{\beta}}$ are the matrix elements of the 2-RDM. Formula (10) relates the spatial overlap integrals with the spin contamination of the wave function.

Likewise, an identical treatment shows that

$$
\begin{aligned}
& {\left[\hat{H},{ }^{p} \hat{S}_{z}\right]=0,} \\
& {\left[\hat{H},{ }^{p} \hat{S}^{2}\right] \neq 0,} \\
& {\left[\hat{H},{ }^{p} \hat{\Omega}\right] \neq 0,}
\end{aligned}
$$

where $\hat{H}$ is the electronic Hamiltonian of the $N$-electron system, which, as is well known, for clamped nuclei can be formulated as

$$
\begin{aligned}
\hat{H}= & \sum_{\sigma} \sum_{i^{\sigma} j^{\sigma}} h_{j^{\sigma}}^{i^{\sigma}} a_{i^{\sigma}}^{\dagger} a_{j^{\sigma}}+\frac{1}{2} \sum_{\sigma} \sum_{i^{\sigma} j^{\sigma} k^{\sigma} l^{\sigma}} R_{j^{\sigma} l^{\sigma}}^{i^{\sigma} k^{\sigma}} a_{i^{\sigma}}^{\dagger} a_{k^{\sigma}}^{\dagger} a_{l^{\sigma}} a_{j^{\sigma}} \\
& +\sum_{\sigma} \sum_{i^{\sigma} j^{\tilde{\sigma}} k^{\sigma} l^{\bar{\sigma}}} R_{j^{\sigma} \bar{\sigma}^{\sigma}}^{i^{\sigma} k^{\bar{\sigma}}} a_{i^{\sigma}}^{\dagger} a_{k^{\bar{\sigma}}}^{\dagger} a_{l^{\sigma}} a_{j^{\sigma}} \quad(\sigma=\alpha, \beta) .
\end{aligned}
$$

In Eq. (14), $\bar{\sigma}$ is the spin conjugate of the spin coordinate $\sigma, h_{j}^{i}$ are the elements of the one-electron integral matrix (the sum of electron kinetic energy and electron-nucleus potential energy), and $R_{j l}^{i k}=\langle i j \mid k l\rangle$ mean the elements of the two-electron repulsion one (expressed in the $\langle 11 \mid 22\rangle$ convention) for the $2 K$ spin-orbital basis set $\left\{i_{1}^{\alpha}, i_{2}^{\alpha} \ldots i_{K}^{\alpha}, i_{1}^{\beta}, i_{2}^{\beta} \ldots i_{K}^{\beta}\right\}$.

For $N$-electron DOCI wave functions, the Hamiltonian expressed in Eq. (14) can be substituted by the effective and simpler
Hamiltonian $\hat{H}^{e f f}$

$$
\begin{aligned}
\hat{H}^{e f f}= & \sum_{\sigma} \sum_{i^{\sigma}} h_{i^{\sigma}}^{i^{\sigma}} a_{i^{\sigma}}^{\dagger} a_{i^{\sigma}}+\frac{1}{2} \sum_{\sigma} \sum_{i^{\sigma} \neq j^{\sigma}} R_{i^{\sigma} j^{\sigma}}^{i^{\sigma} j^{\sigma}} a_{i^{\sigma}}^{\dagger} a_{j^{\sigma}}^{\dagger} a_{j^{\sigma}} a_{i^{\sigma}} \\
& +\sum_{\sigma} \sum_{i^{\sigma} \neq j^{\tilde{\sigma}}} R_{i^{\sigma} j^{\sigma}}^{i^{\sigma} j^{\tilde{\sigma}}} a_{i^{\sigma}}^{\dagger} a_{j^{\sigma}}^{\dagger} a_{j^{\sigma} \sigma} a_{i^{\sigma}}+\frac{1}{2} \sum_{\sigma} \sum_{i^{\sigma} \neq j^{\sigma}} R_{j^{\sigma} i^{\sigma}}^{i^{\sigma} \sigma^{\sigma}} a_{i^{\sigma}}^{\dagger} a_{j^{\sigma}}^{\dagger} a_{i^{\sigma}} a_{j^{\sigma}} \\
& +\sum_{\sigma} \sum_{i^{\sigma} \neq j^{\sigma}} R_{j^{\sigma} j^{\sigma}}^{i^{\sigma} i^{\sigma}} a_{i^{\sigma}}^{\dagger} a_{i^{\sigma}}^{\dagger} a_{j^{\tilde{\sigma}}} a_{j^{\sigma}} \quad(\sigma=\alpha, \beta)
\end{aligned}
$$

which contains identical information than the more general Hamiltonian, $\hat{H}$, but requires much less terms. ${ }^{22}$ Contrarily to the general Hamiltonian, the effective Hamiltonian, $\hat{H}^{e f f}$, commutes not only with the ${ }^{p} \hat{S}_{z}$ operator but also with the ${ }^{p} \hat{S}^{2}$ and ${ }^{p} \hat{\Omega}$ ones. In fact, the projections of both Hamiltonians, $\hat{H}$ and $\hat{H}^{\text {eff }}$, over the Hilbert space of doubly occupied $N$-electron Slater determinants lead to the same result. According to the formulation of this effective Hamiltonian, only the diagonal elements ${ }^{1} D_{i^{\sigma}}^{i^{\sigma}}$ of the 1-RDM and the matrix elements ${ }^{2} D_{i^{\sigma} j^{\sigma}}^{i^{\sigma} j^{\sigma}},{ }^{2} D_{i^{\sigma} j^{\sigma^{\sigma}}}^{i^{\sigma} j^{\sigma}},{ }^{2} D_{j^{\sigma} j^{\sigma}}^{i^{\sigma} j^{\sigma}}$, and ${ }^{2} D_{j^{\sigma} j^{\sigma^{\sigma}}}^{i^{\sigma^{\sigma}} \tilde{i}^{\tilde{\sigma}}}$ for the 2-RDM need be calculated; the remainder of the elements of the 1-RDM and 2-RDM are zero within the DOCI schemes. Consequently, our variational algorithms will use this effective Hamiltonian, $\hat{H}^{\text {eff }}$, described in Eq. (15).

As has been mentioned in Sec. I, this work deals with RDMs corresponding to DOCI wave functions described by Slater determinants in which all orbitals are doubly occupied. Strictly speaking, a doubly occupied orbital requires that the spatial function of the $\alpha$ - and $\beta$-spin orbitals is identical; however, according to the defined "pairing" between the spin orbitals $i_{j}^{\alpha}$ and $i_{j}^{\beta}, \forall j$, we will use this pseudodouble occupation for these orbitals. Consequently, these kinds of wave functions are eigenfunctions of the operators ${ }^{p} \hat{S}_{z}$, ${ }^{p} \hat{S}^{2}$, and ${ }^{p} \hat{\Omega}$ with a numerical value zero for the three corresponding eigenvalues. Due to the commutation relationships (7)-(9), the RDMs arising from the DOCI wave functions possess identical spin structure as in the orthogonal orbital case as in the nonorthogonal orbital one. Hereafter, we will distinguish between the restricteddoubly occupied-configuration-interaction (RDOCI) wave functions (the orbital functions are orthogonal) and the unrestricteddoubly occupied-configuration-interaction (UDOCI) ones, in which not all orbital functions of the basis set are necessarily orthonormal. ${ }^{27}$ In Sec. III, we report the computational aspects followed in this work to determine variationally the 2-RDM elements corresponding to a DOCI type approximation of the ground state of an $N$-electron system. Likewise, in the Appendix, we formulate all constraints imposed on the elements of the 1- and 2-RDM in the implementation of that method.

\section{COMPUTATIONAL ASPECTS, RESULTS, AND DISCUSSION}

Results arising from the UDOCI procedure, evaluating $\mathrm{N}$ electron wave functions and the corresponding 2-RDMs, have been compared with those arising from its variational version, v2RDMUDOCI, evaluating directly the 2-RDMs by that method and avoiding the explicit calculation of the wave functions. These tests have been performed by numerical determinations of ground state 
energies in several $\mathrm{H}_{4}$ clusters possessing different geometric configurations (see Fig. 1). In that figure, we show the shapes of those clusters as well as the parameters chosen to transform their geometries. We have calculated the potential energy curves (PECs) corresponding to these aggregates and the resulting expectation values of the spin-squared operator, $\left\langle\hat{S}^{2}\right\rangle$, which allow one to assess the spin contamination of their states. The results arising from UDOCI and v2RDM-UDOCI methods have been compared with those obtained from their counterparts RDOCI and v2RDM-RDOCI treatments. We have also studied the influence of the diverse $N$-representability conditions in the cases of variational schemes. We have used the atomic basis sets STO-3G, 6-31G, and 6-311G, which provide an affordable computational cost and allow to study the dependence of our results on the basis set size. The standard one- and twoelectron integrals have been obtained from the PSI3.4 package ${ }^{47}$ in the canonical molecular orbital, restricted and unrestricted, basis sets. The RDOCI and UDOCI wave function-based results have been obtained from modified versions of the direct determinant-FCI algorithm reported in Refs. 48 and 49, which build and diagonalize the projection of the Hamiltonian in the seniority-zero space. Several local minima appear in the orbital optimization task; consequently, we have extended the simulated annealing procedure reported in Ref. 28 to search RDOCI and UDOCI energy global minima, enabling to perform orbital rotations of the $\alpha$ - and $\beta$-basis sets independently. This technique yields restricted and unrestricted versions of the basis sets by optimizing the energy (OPTE). In Fig. 2, we show the optimized STO-3G basis set orbitals for the $P_{4}\left(D_{2 h}\right)$ $B$ cluster upon variation of the $b$ side length. As can be seen in that figure, the resulting orbitals break spatial symmetry and in the unrestricted case, the $\alpha$ - and $\beta$-orbitals localize adopting distinct shapes.

For the two-electron-reduced-density-matrix-based variational calculations, we have progressively incorporated all constraints shown in the Appendix, particularly the two- and three-index $N$-representability conditions, to a modified $\operatorname{SDPA}^{9,26}$ using the effective Hamiltonian reported in Eq. (15). In this kind of calculations, we used the semidefinite programing algorithm code
SDPA 7.3.8, ${ }^{26}$ which solves semidefinite problems at several precision levels by means of the Mehrotra-type predictor-corrector primal-dual interior-point method, providing ground-state energies and the corresponding 2-RDMs. We programed the variational method as a dual problem in the SDPA code. The sparse structure of the ${ }^{2} P,{ }^{2} Q,{ }^{2} G, T 1$, and $T 2$ matrices, within the RDOCI and UDOCI frameworks, gives rise to a scaling of the v2RDMUDOCI method nearly identical to that of the v2RDM-RDOCI one: ${ }^{23} O\left(K^{3}\right)$ for the two-index conditions and $O\left(K^{4}\right)$ for the threeindex ones. Consequently, from a computational point of view, the unrestricted treatment in the variational reduced density matrix framework does not entail any significant increase in computational effort.

In Fig. 3, we show the PECs corresponding to the ground states of the above mentioned nuclear geometric configurations of the $\mathrm{H}_{4}$ cluster. These curves have been obtained from different methods within the DOCI methodology (RDOCI, UDOCI, v2RDM-RDOCI, and v2RDM-UDOCI treatments). In the case of variational methods, their results have been obtained under the PQGT1T2 conditions. We have also included in that figure the results arising from the restricted Hartree-Fock (RHF) and unrestricted HartreeFock (UHF) methods as well as those of the FCI one in order to carry out a suitable comparison of all results. As can be seen in Fig. 3, the v2RDM-RDOCI and RDOCI curves are, for all configurations, nearly coincident. Similarly, the v2RDM-UDOCI and UDOCI curves present identical performance; they also coincide with each other. An inherent feature of all unrestricted treatments is the appearance of spin contamination; this feature has been quantified by the $\left\langle\hat{S}^{2}\right\rangle$ quantity, whose values along the PECs have been shown in the bottom panels in Fig. 3. The UDOCI and v2RDMUDOCI methods present lower $\left\langle\hat{S}^{2}\right\rangle$ values than those corresponding to the UHF one. In the $S_{4}\left(D_{4 h}\right)$ system, we must highlight that all of its curves, except the RHF one, show a similar shape, although those resulting from the UDOCI and v2RDM-UDOCI methods are the closest to the FCI one. This behavior is also observed in the $P_{4}\left(D_{2 h}\right)$-A cluster, in which the UDOCI and v2RDM-UDOCI curves are quite parallel to the FCI one, improving the UHF result. The
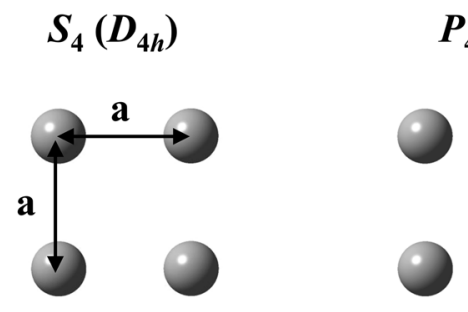

$P_{4}\left(D_{2 h}\right)-\mathrm{A}$

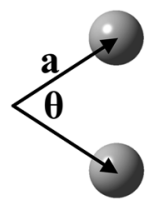

Rhomboid

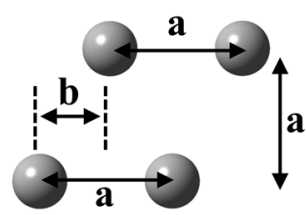

$P_{4}\left(D_{2 h}\right)-\mathrm{B}$

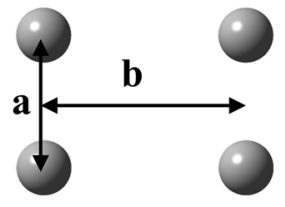

Twisted

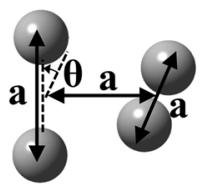

FIG. 1. Geometric arrangements of the $\mathrm{H}_{4}$ aggregates studied in this work and parameters used for their characterization. Square $S_{4}\left(D_{4 h}\right)$ : a variable. Rectangular (in a ring) $P_{4}\left(D_{2 h}\right)-A: a=0.748$ $\AA$ A, $\theta$ variable. Rectangular (stretched) $P_{4}\left(D_{2 h}\right)$-B: a $=1.058 \AA$, b variable. Rhombus: $\mathrm{a}=1.497 \AA$, $\mathrm{b}$ variable. Rhomboid: $a=1.058 \AA$, $b$ variable. Twisted: $\mathrm{a}=1.058 \AA, \theta$ variable. 


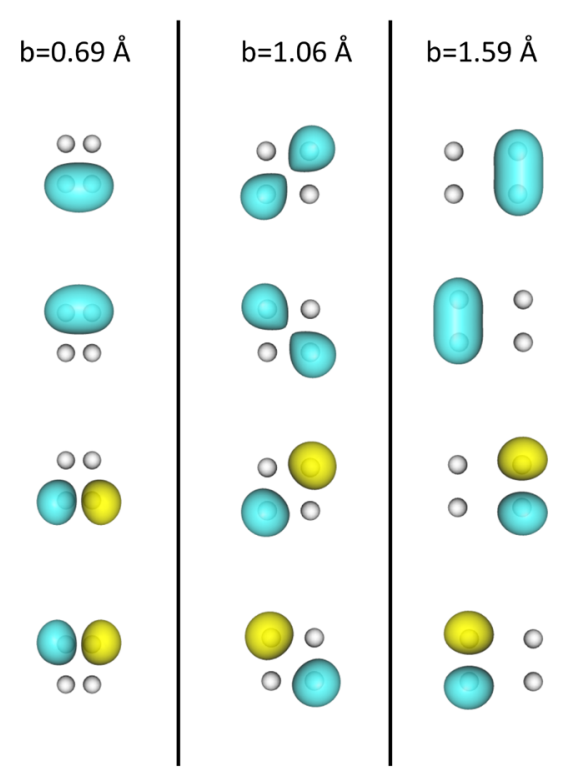

(a)

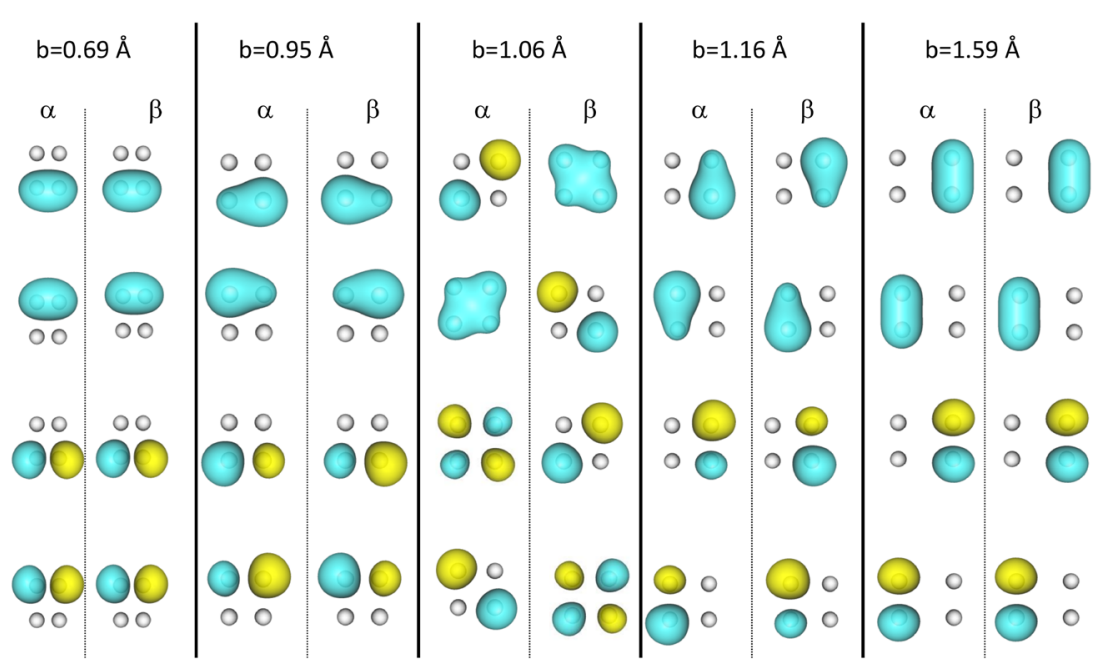

(b)
FIG. 2. Isosurfaces of the (a) restricted and (b) unrestricted optimized orbitals (OPTE) arising from STO-3G basis sets for the $P_{4}\left(D_{2 h}\right)$-B cluster of $\mathrm{H}_{4}$ at several geometries. Positive values are indicated in yellow and negative values in pastel blue. The isovalues are \pm 0.12 a.u.
$P_{4}\left(D_{2 h}\right)$-B system presents an unphysical cusp in the RDOCI and v2RDM-RDOCI methods, which has also been reported by other authors, ${ }^{50}$ but it does not appear in the UDOCI and v2RDM-UDOCI ones. Likewise, the Rhombus and Rhomboid PECs also show similar shapes for all methods, and in the Twisted system, which corresponds to a nonplanar configuration, one can observe again superior closeness to the FCI values in the UDOCI procedures than in the RDOCI ones. In order to analyze globally all the information contained in the PECs, we have given in Table I the maximum absolute errors (MAEs) of the DOCI methodology energy values with respect to the FCI ones. Table I also reports the nonparallelity errors (NPEs), i.e., the differences between the maximum and minimum deviations of DOCI energies from the FCI energy. Very similar MAE and NPE values have been obtained for the RDOCI and v2RDMRDOCI procedures and, similarly, for the UDOCI with respect to the v2RDM-UDOCI one, what points out the suitability of the variational approximations under the PQGT1T2 N-representability conditions. Moreover, as can be seen in that table, the MAE and NPE values of the UDOCI and v2RDM-UDOCI methods are markedly lower (one third or less) than those resulting from their RDOCI and v2RDM-RDOCI counterparts, showing an important improvement in the description of this kind of systems. In Table II, we show the maximum root-mean-square deviation (RMSD) of the 1-RDMs in the ground state of the $\mathrm{H}_{4}$ studied clusters, obtained from the v2RDM-RDOCI and v2RDM-UDOCI methods with respect to the RDOCI and UDOCI results, imposing the PQGT1T2 conditions. As 

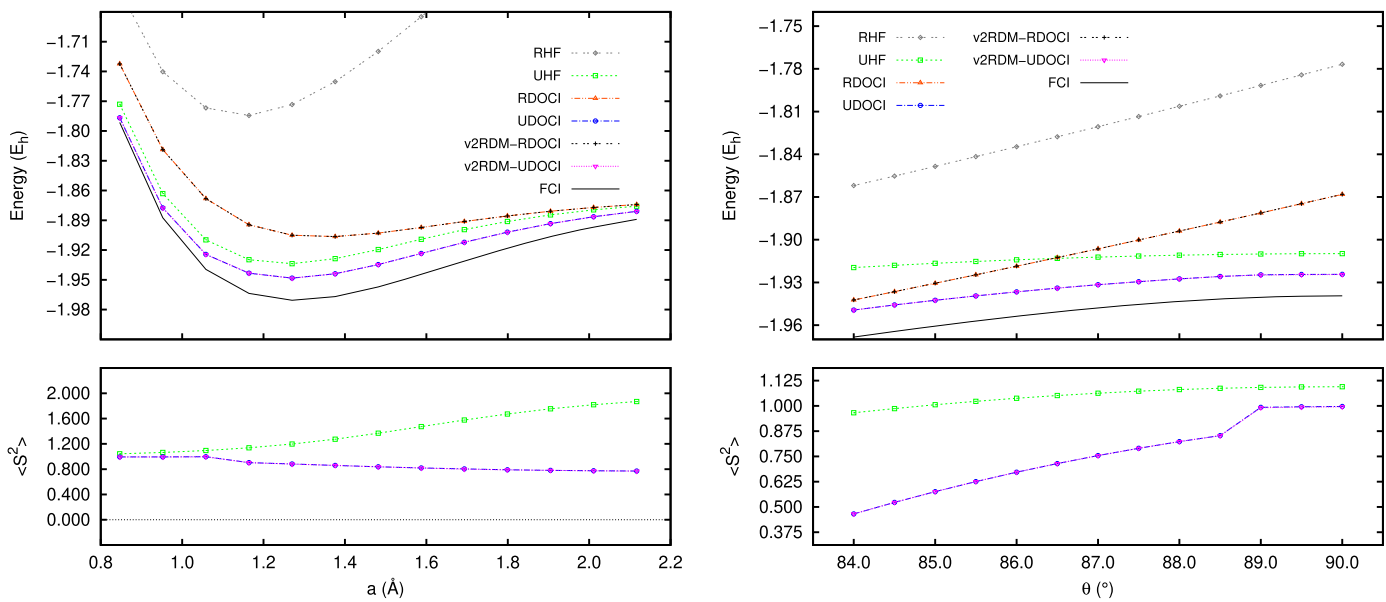

(a)

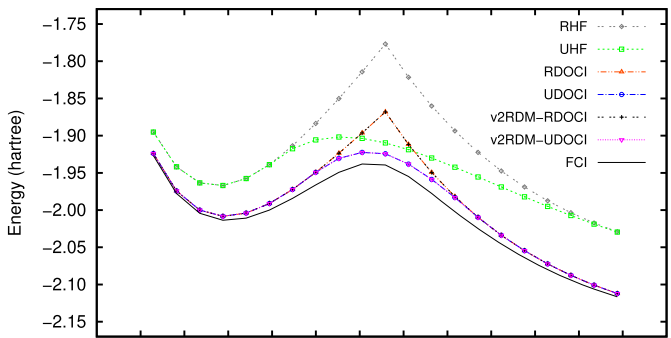

(b)

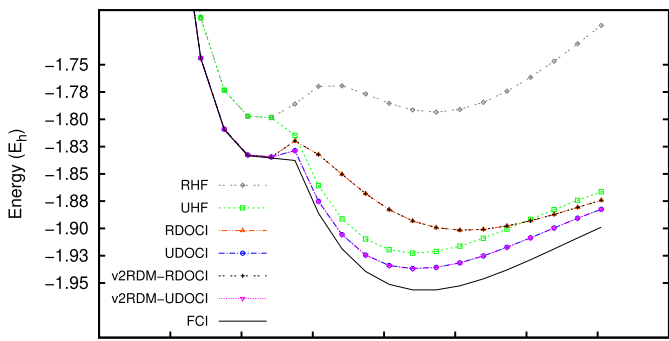

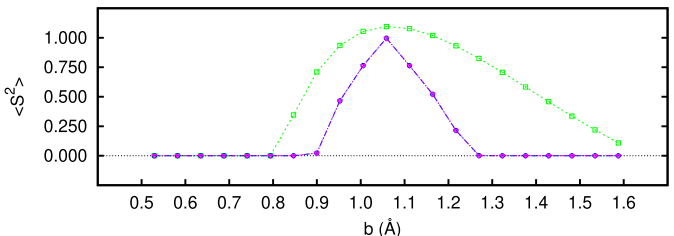

(c)
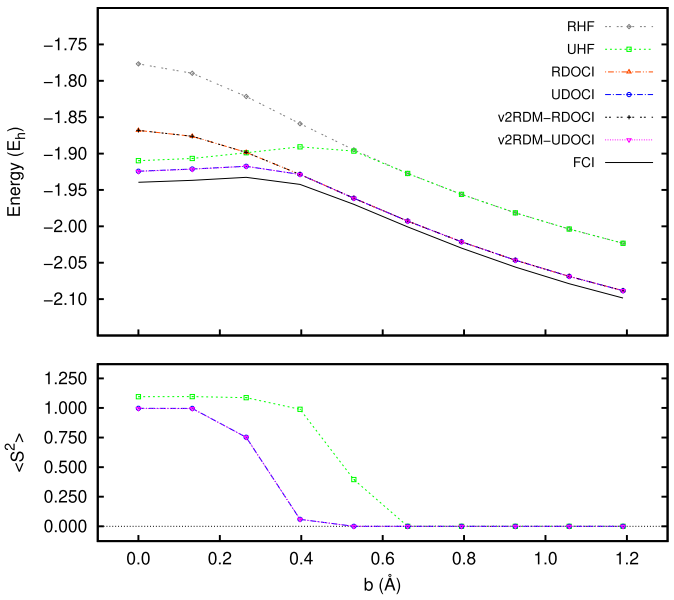

(e)

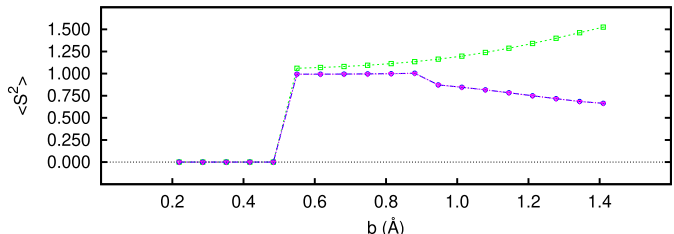

(d)
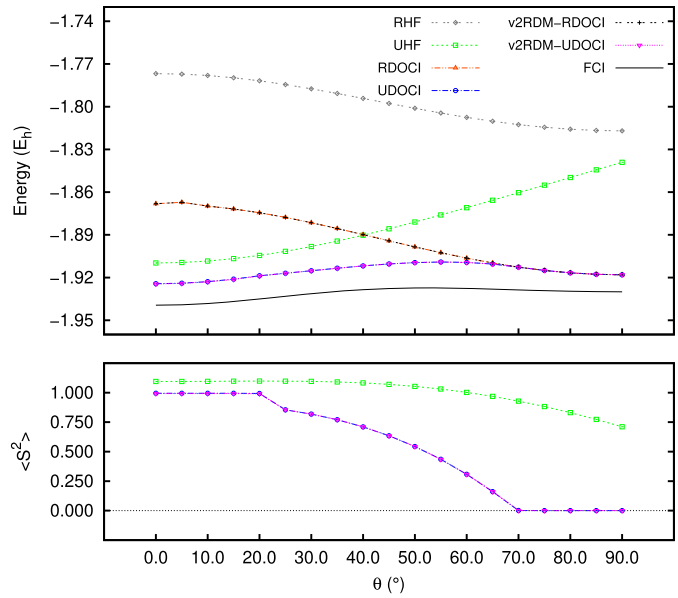

(f)

FIG. 3. Ground-state potential energy curves of the $\mathrm{H}_{4}$ cluster at the nuclear conformations: (a) $S_{4}\left(D_{4 h}\right),\left(\right.$ b) $P_{4}\left(D_{2 h}\right)-\mathrm{A}$, (c) $P_{4}\left(D_{2 h}\right)-\mathrm{B}$, (d) Rhombus, (e) Rhomboid, and (f) Twisted. Results were obtained from the STO-3G atomic basis set transformed into the orbital set which minimizes the energy (OPTE) (RDOCI and UDOCI methods). The variational method results (V2RDM-RDOCI and v2RDM-UDOCI) arise from the PQGT1T2 conditions. The bottom panels show $\left\langle S^{2}\right\rangle$ values for UHF, UDOCI, and V2RDM-UDOCI methods. 
TABLE I. Maximum absolute errors (MAEs) and nonparallelity errors (NPEs) (in $m E_{h}$ ) of the ground state potential energy curves of $\mathrm{H}_{4}$ clusters calculated by RDOCl, v2RDM-RDOCI, UDOCl, and V2RDM-UDOCI methods with respect to the FCl values. The variational methods arise from the PQGT1T2 conditions and optimized orbitals (OPTE) from the STO-3G atomic basis set.

\begin{tabular}{|c|c|c|c|c|c|c|c|c|}
\hline \multirow[b]{2}{*}{ System } & \multicolumn{2}{|c|}{ RDOCI } & \multicolumn{2}{|c|}{ v2RDM-RDOCI } & \multicolumn{2}{|c|}{ UDOCI } & \multicolumn{2}{|c|}{ v2RDM-UDOCI } \\
\hline & MAE & NPE & MAE & NPE & MAE & NPE & MAE & NPE \\
\hline$S_{4}\left(D_{4 h}\right)$ & 71.2636 & 56.3109 & 71.2627 & 56.3100 & 23.0230 & 18.3385 & 22.9601 & 18.3364 \\
\hline$P_{4}\left(D_{2 h}\right)-\mathrm{A}$ & 71.2636 & 45.2320 & 71.2627 & 45.2318 & 18.9561 & 3.8491 & 18.9563 & 4.0019 \\
\hline$P_{4}\left(D_{2 h}\right)-\mathrm{B}$ & 71.2636 & 68.3892 & 71.2627 & 68.3884 & 18.8915 & 16.0164 & 18.8791 & 16.0041 \\
\hline Rhombus & 71.2636 & 71.0390 & 71.2627 & 71.0384 & 21.0857 & 20.8611 & 21.0277 & 20.8034 \\
\hline Rhomboid & 71.2636 & 63.2501 & 71.2627 & 63.2518 & 15.5174 & 7.5035 & 15.3550 & 7.3438 \\
\hline Twisted & 71.9697 & 60.0276 & 71.9688 & 60.0268 & 18.2475 & 6.3053 & 18.2424 & 6.3005 \\
\hline
\end{tabular}

can be observed, very low differences have been found, although the maximum RMSD values are greater in the v2RDM-UDOCI method than in the v2RDM-RDOCI one; we interpret this fact in terms of the basis-set dependence of the strength of the $N$-representability conditions within the DOCI framework. 10,11,22,23,25

We have assessed the influence of the positive semidefiniteness constraint condition sets $P Q G, P Q G T 1$, and $P Q G T 1 T 2$, imposed in the variational methods, on our results. Table III gives values of MAE and NPE quantities obtained from the v2RDM-RDOCI and v2RDM-UDOCI methods with respect to the RDOCI and UDOCI ones, respectively, for all geometric conformations of the $\mathrm{H}_{4}$ cluster studied in this work. In this table, one can observe low MAE and NPE values arising from the v2RDM-RDOCI method, what is in agreement with the overlaps of their PECs and those of RDOCI method shown in Fig. 3. The sequence of $N$-representability condition sets $P Q G, P Q G T 1$, and $P Q G T 1 T 2$ yields MAE and NPE values converging to nearly zero for the v2RDM-RDOCI method. Nevertheless, the v2RDM-UDOCI method presents higher MAE and NPE values, what agrees with the numerical values reported in Table II. However, the consequences of these deviations do not become too significant, mainly when one uses the most demanding constraint condition set PQGT1T2.

In Table IV, we have given the results obtained from numerical determinations implemented on the square $\mathrm{H}_{4}$ cluster $S_{4}\left(D_{4 h}\right)$

TABLE II. Maximum root-mean-square deviation (RMSD) of the 1-RDMs in the ground state of $\mathrm{H}_{4}$ clusters calculated by the V2RDM-RDOCI and V2RDM-UDOCl methods imposing the PQGT1T2 conditions with respect to the RDOCI and UDOCI results, using optimized orbitals (OPTE) from the STO-3G atomic basis set.

\begin{tabular}{lcc}
\hline \hline & \multicolumn{2}{c}{ RMSD } \\
\cline { 2 - 3 } System & v2RDM-RDOCI & v2RDM-UDOCI \\
\hline$S_{4}\left(D_{4 h}\right)$ & $6.02 \times 10^{-7}$ & $6.89 \times 10^{-4}$ \\
$P_{4}\left(D_{2 h}\right)-\mathrm{A}$ & $8.91 \times 10^{-7}$ & $1.94 \times 10^{-4}$ \\
$P_{4}\left(D_{2 h}\right)-\mathrm{B}$ & $7.92 \times 10^{-7}$ & $1.92 \times 10^{-4}$ \\
Rhombus & $3.87 \times 10^{-5}$ & $3.10 \times 10^{-4}$ \\
Rhomboid & $3.00 \times 10^{-6}$ & $1.96 \times 10^{-4}$ \\
Twisted & $8.40 \times 10^{-7}$ & $2.06 \times 10^{-4}$ \\
\hline \hline
\end{tabular}

in order to assess the atomic basis set dependence of this methodology. In that table, we show the energy differences, $\Delta \mathrm{E}$, arising from the RDOCI, v2RDM-RDOCI, UDOCI, and v2RDM-UDOCI methods with respect to the FCI values in the atomic basis sets STO$3 \mathrm{G}, 6-31 \mathrm{G}$, and 6-311G. We also show values of $\left\langle\hat{S}^{2}\right\rangle$ assessing the spin contamination resulting from the UDOCI and v2RDM-UDOCI methods using these atomic basis sets. A survey of these results again reveals a superior behavior of the unrestricted methods with respect

TABLE III. Maximum absolute errors (MAEs) and nonparallelity errors (NPEs) (in $m E_{h}$ ) of the ground state potential energy curves of $\mathrm{H}_{4}$ clusters calculated by the v2RDM-RDOCl and v2RDM-UDOCl methods imposing the PQG, PQGT1, and $P Q G T 1 T 2$ conditions with respect to the RDOCl and UDOCl results, using optimized orbitals (OPTE) from the STO-3G atomic basis set.

\begin{tabular}{|c|c|c|c|c|c|}
\hline \multirow[b]{2}{*}{ System } & \multirow[b]{2}{*}{ Conditions } & \multicolumn{2}{|c|}{ v2RDM-RDOCI } & \multicolumn{2}{|c|}{ v2RDM-UDOCI } \\
\hline & & MAE & NPE & MAE & NPE \\
\hline \multirow[t]{3}{*}{$S_{4}\left(D_{4 h}\right)$} & $P Q G$ & 0.1178 & 0.1175 & 3.9670 & 3.7026 \\
\hline & PQGT1 & 0.1177 & 0.1174 & 1.7466 & 1.7124 \\
\hline & $P Q G T 1 T 2$ & 0.0009 & 0.0009 & 0.1609 & 0.1338 \\
\hline \multirow{3}{*}{$P_{4}\left(D_{2 h}\right)-\mathrm{A}$} & $P Q G$ & 0.0277 & 0.0092 & 3.9671 & 3.8449 \\
\hline & $P Q G T 1$ & 0.0256 & 0.0090 & 1.7466 & 1.7204 \\
\hline & PQGT1T2 & 0.0009 & 0.0002 & 0.1624 & 0.1544 \\
\hline \multirow[t]{3}{*}{$P_{4}\left(D_{2 h}\right)-\mathrm{B}$} & $P Q G$ & 0.0277 & 0.0255 & 3.9670 & 3.9648 \\
\hline & PQGT1 & 0.0255 & 0.0248 & 1.7466 & 1.7459 \\
\hline & $P Q G T 1 T 2$ & 0.0009 & 0.0008 & 0.1609 & 0.1608 \\
\hline \multirow[t]{3}{*}{ Rhombus } & $P Q G$ & 1.3998 & 1.3777 & 4.9160 & 4.8157 \\
\hline & PQGT1 & 0.1021 & 0.0961 & 2.0916 & 2.0806 \\
\hline & $P Q G T 1 T 2$ & 0.0372 & 0.0099 & 0.2291 & 0.2288 \\
\hline \multirow[t]{3}{*}{ Rhomboid } & $P Q G$ & 0.7748 & 0.7505 & 3.9763 & 3.6714 \\
\hline & $P Q G T 1$ & 0.0936 & 0.0863 & 1.7466 & 1.7393 \\
\hline & $P Q G T 1 T 2$ & 0.0034 & 0.0030 & 0.1624 & 0.1616 \\
\hline \multirow[t]{3}{*}{ Twisted } & $P Q G$ & 0.1637 & 0.1525 & 4.0000 & 3.9881 \\
\hline & PQGT1 & 0.0628 & 0.0606 & 1.7515 & 1.7399 \\
\hline & $P Q G T 1 T 2$ & 0.0009 & 0.0009 & 0.1672 & 0.1672 \\
\hline
\end{tabular}


TABLE IV. Energy differences, $\triangle \mathrm{E}$ (in $m E_{h}$ ), of diverse methods with respect to the $\mathrm{FCl}$ ones and spin contamination and $\left\langle\hat{S}^{2}\right\rangle$ (in the UDOCl and v2RDM-UDOCl approaches) in the ground state of the $\mathrm{H}_{4}$ cluster (conformation $S_{4}\left(D_{4 h}\right)$ with a $=1.058 \AA$ ). Results correspond to optimized orbitals (OPTE) arising from STO-3G, 6-31G and 6-311G atomic basis sets, imposing the PQGT1T2 conditions (in the v2RDM-RDOCl and v2RDM-UDOCI procedures).

\begin{tabular}{lccccccc}
\hline \hline & \multicolumn{9}{c}{$\Delta \mathrm{E}$} & & \multicolumn{2}{c}{$\left\langle\hat{S}^{2}\right\rangle$} \\
\cline { 2 - 4 } \cline { 7 - 8 } Basis & RDOCI & v2RDM-RDOCI & UDOCI & v2RDM-UDOCI & & UDOCI & v2RDM-UDOCI \\
\hline STO-3G & 71.2636 & 71.2627 & 15.1152 & 14.9544 & & 0.9967 & 0.9926 \\
6-31G & 46.1281 & 46.1246 & 13.1764 & 13.1658 & & 0.9063 & 0.9063 \\
6-311G & 45.6988 & 45.6935 & 14.6273 & 14.6171 & & 0.9251 & 0.9251 \\
\hline \hline
\end{tabular}

to the restricted ones in terms of energy. Moreover, the differences arising from the unrestricted methods vary very slightly from one basis set to another and a similar comment can be made in relation to the $\left\langle\hat{S}^{2}\right\rangle$ values obtained from those methods.

\section{CONCLUSIONS}

In this work, we have proposed a procedure based on the use of unrestricted orbitals to determine variationally the two-electron reduced density matrices of $N$-electron systems described by doubly occupied-configuration-interaction wave functions. We have evaluated energies and values of the $\left\langle\hat{S}^{2}\right\rangle$ quantity in six different geometric conformations of the $\mathrm{H}_{4}$ cluster by means of progressive application of the well-known positive semidefiniteness constraint condition sets $P Q G, P Q G T 1$, and PQGT1T2. The results found have been compared with those obtained from their counterpart treatments in the restricted orbital approach and with those predicted by conventional (restricted and unrestricted) wave functionbased procedures. An assessment of all numerical results shows higher closeness to the exact energies in the unrestricted treatments than in the restricted ones, although the former entail a spin contamination. We also show the suitability of the use of the $N$-representability PQGT1T2 constraint conditions in all studied variational methods.

\section{ACKNOWLEDGMENTS}

The authors acknowledge financial support from the Universidad de Buenos Aires (Grant No. 20020150100157BA), the Consejo Nacional de Investigaciones Científicas y Técnicas (Grant Nos. PIP 11220130100377CO, PIP 11220130100311CO, and 2013-1401PCB), and the Agencia Nacional de Promoción Científica y Tecnológica (Grant No. PICT-201-0381), Argentina. E.R. gratefully acknowledges a doctoral fellowship granted by the Consejo Nacional de Investigaciones Científicas y Técnicas.

\section{APPENDIX: $\boldsymbol{N}$-REPRESENTABILITY CONSTRAINTS USED IN THE VARIATIONAL DETERMINATION OF 2-RDM ARISING FROM RDOCI AND UDOCI WAVE FUNCTIONS}

In this section, we summarize the $N$-representability constraints imposed to the RDMs in the variational determination of the 2-RDM performed in this work

$$
\begin{aligned}
& \sum_{i^{\sigma}}{ }^{1} D_{i^{\sigma}}^{i^{\sigma}}=\frac{N}{2} \quad(\sigma=\alpha, \beta), \\
& \sum_{i^{\sigma}} \sum_{j^{\sigma}}{ }^{2} D_{i^{\sigma} j^{\sigma}}^{i^{\sigma} j^{\sigma}}=\left(\begin{array}{c}
\frac{N}{2} \\
2
\end{array}\right) \quad(\sigma=\alpha, \beta), \\
& \sum_{i^{\sigma}} \sum_{j^{\tilde{\sigma}}}{ }^{2} D_{i^{\sigma} j^{\sigma} j^{\sigma} j^{\tilde{\sigma}}}=\frac{\left(\frac{N}{2}\right)^{2}}{2} \quad(\sigma=\alpha, \beta), \\
& { }^{1} D_{i^{\sigma}}^{i^{\sigma}}=\frac{2}{N-1}\left[\sum_{j^{\sigma}}{ }^{2} D_{i^{\sigma} j^{\sigma}}^{i^{\sigma} j^{\sigma}}+\sum_{j^{\tilde{\sigma}}}{ }^{2} D_{i^{\sigma} j^{i^{\sigma}}}^{i^{\sigma} \tilde{j}^{\tilde{\sigma}}}\right] \quad(\sigma=\alpha, \beta), \\
& \sum_{\sigma} \sum_{i^{\sigma}}{ }^{2} D_{i^{\sigma} i^{\tilde{\sigma}}}^{i^{\sigma} i^{\tilde{\sigma}}}=\frac{N}{2} \quad(\sigma=\alpha, \beta) .
\end{aligned}
$$

Equations (A1)-(A3) indicate the normalization conditions of the different spin blocks for the 1- and 2-RDMs arising from the RDOCI and UDOCI schemes. Equation (A4) constitutes the contraction relationship between the 2- and 1-RDMs. Equation (A5) is obtained from Eq. (6) for the case $\left\langle{ }^{p} \hat{\Omega}\right\rangle=0$, which is a necessary condition for the RDOCI and UDOCI wave functions. Apart from these basic conditions, the 1-RDM and the hole first-order reduced density matrix, ${ }^{1} Q$, must be positive semidefinite, that is, ${ }^{1} D_{j^{\sigma}}^{i^{\sigma}}=\left\langle\Psi\left|a_{i^{\sigma}}^{\dagger} a_{j^{\sigma}}\right| \Psi\right\rangle \geq 0$ and ${ }^{1} Q_{j^{\sigma}}^{i^{\sigma}}$ $=\left\langle\Psi\left|a_{j^{\sigma}} a_{i^{\sigma}}^{\dagger}\right| \Psi\right\rangle \geq 0$, and for RDOCI and UDOCI wave functions, both of them are diagonal matrices. Consequently, their matrix elements satisfy

$$
{ }^{1} D_{i^{\sigma}}^{i^{\sigma}}=\left\langle\Psi\left|a_{i^{\sigma}}^{\dagger} a_{i^{\sigma}}\right| \Psi\right\rangle \geq 0 \quad(\sigma=\alpha, \beta),
$$

and

$$
{ }^{1} Q_{i^{\sigma}}^{i^{\sigma}}=\left\langle\Psi\left|a_{i^{\sigma}} a_{i^{\sigma}}^{\dagger}\right| \Psi\right\rangle \geq 0 \quad(\sigma=\alpha, \beta) .
$$

The $P$ condition requires that the matrix ${ }^{2} P_{j^{\sigma_{1}} l^{\sigma_{2}}}^{i_{1} k_{2}^{\sigma_{2}}}=$ $\left\langle\Psi\left|a_{i^{\sigma_{1}}}^{\dagger} a_{k^{\sigma_{2}}}^{\dagger} a_{l^{\sigma_{2}}} a_{j^{\sigma_{1}}}\right| \Psi\right\rangle \geq 0 \quad\left(\sigma_{1}, \sigma_{2}=\alpha, \beta\right)$. For RDOCI and UDOCI cases, this matrix is decomposed into the spin blocks

$$
{ }^{2} P_{j^{\sigma} j^{\tilde{\sigma}}}^{i^{\sigma} \dot{\sigma}^{\tilde{\sigma}}}=2^{2} D_{j^{\sigma} j^{i^{\sigma}}}^{i^{\sigma} \tilde{\sigma}^{\tilde{\sigma}}} \quad \text { and } \quad{ }^{2} P_{i^{\sigma} j^{\sigma}}^{i^{\sigma} j^{\tilde{\sigma}}}=2^{2} D_{i^{\sigma} j^{\tilde{\sigma}}}^{i^{\sigma} j^{\tilde{\sigma}}} \quad(\sigma=\alpha, \beta),
$$

and 


$$
{ }^{2} P_{i^{\sigma} j^{\sigma}}^{i^{\sigma} \sigma^{\sigma}}=2{ }^{2} D_{i^{\sigma} j^{\sigma}}^{i^{\sigma} j^{\sigma}} \quad(i \neq j, \sigma=\alpha, \beta) .
$$

Each spin block constitutes a positive semidefinite matrix.

Similarly, the $Q$ condition means that the matrix ${ }^{2} Q_{j^{\sigma_{1}} l^{\sigma_{2}}}^{i_{1} k_{2}^{\sigma_{2}}}$ $=\left\langle\Psi\left|a_{j^{\sigma_{1}}} a_{l^{\sigma_{2}}} a_{k^{\sigma_{2}}}^{\dagger} a_{i^{\sigma_{1}}}^{\dagger}\right| \Psi\right\rangle \geq 0 \quad\left(\sigma_{1}, \sigma_{2}=\alpha, \beta\right)$. For the DOCI frameworks, this matrix is constituted by the spin blocks

$$
\begin{aligned}
& { }^{2} Q_{j^{\sigma} j^{i^{\sigma}}}^{i^{\sigma} \tilde{\sigma}^{\tilde{\sigma}}}=2{ }^{2} D_{j^{\sigma} j^{i^{\sigma}}}^{i^{\sigma_{i}} i^{\tilde{\sigma}}}+\left(1-{ }^{1} D_{i^{\sigma}}^{i^{\sigma}}-{ }^{1} D_{i^{\tilde{\sigma}}}^{i^{\tilde{\sigma}^{\sigma}}}\right) \delta_{i j} \quad(\sigma=\alpha, \beta) \quad \text { and } \\
& { }^{2} Q_{i^{\sigma} j^{\tilde{\sigma}}}^{i^{\sigma} j^{\tilde{\sigma}}}=2{ }^{2} D_{i^{\sigma} j^{\tilde{\sigma}}}^{i^{\sigma} j^{\tilde{\sigma}}}+1-{ }^{1} D_{i^{\sigma}}^{i^{\sigma}}-{ }^{1} D_{j^{\tilde{\sigma}}}^{j^{\tilde{\sigma}}} \quad(i \neq j, \sigma=\alpha, \beta),
\end{aligned}
$$

and

$$
{ }^{2} Q_{i^{\sigma} j^{\sigma}}^{i^{\sigma} j^{\sigma}}=1-{ }^{1} D_{i^{\sigma}}^{i^{\sigma}}-{ }^{1} D_{j^{\sigma}}^{j^{\sigma}}+2^{2} D_{i^{\sigma} j^{\sigma}}^{i^{\sigma} j^{\sigma}} \quad(i \neq j, \sigma=\alpha, \beta)
$$

which must be again positive semidefinite.

The $G$ condition is defined in terms of the particle-hole matrix, that is, ${ }^{2} G_{j^{\sigma_{2}} k^{\sigma_{4}}}^{i^{\sigma_{1} \sigma_{3}}}=\left\langle\Psi\left|a_{i^{\sigma_{1}}}^{\dagger} a_{l^{\sigma_{3}}} a_{k^{\sigma_{4}}}^{\dagger} a_{j^{\sigma_{2}}}\right| \Psi\right\rangle \geq 0 \quad\left(\sigma_{1}, \sigma_{2}, \sigma_{3}, \sigma_{4}=\alpha, \beta\right)$. The elements of this matrix are zero if the spin-coordinate distribution satisfies the condition $\left(\sigma_{1}+\sigma_{4} \neq \sigma_{2}+\sigma_{3}\right)$, what leads to the following spin blocks for the DOCI frameworks:

$$
\begin{aligned}
& \left(\begin{array}{cc}
{ }^{2} G_{j^{\alpha} j^{\alpha}}^{i^{\alpha} j^{\alpha}} & { }^{2} G_{j^{\alpha} j^{\alpha}}^{i^{\beta} \beta^{\beta}} \\
{ }^{2} G_{j^{\beta} j^{\beta}}^{i^{\alpha} i^{\alpha}} & { }^{2} G_{j^{\beta} j^{\beta}}^{i^{\beta} \beta^{\beta}}
\end{array}\right)=\left(\begin{array}{cc}
2{ }^{2} D_{i^{\alpha} j^{\alpha}}^{i^{\alpha} j^{\alpha}}+{ }^{1} D_{i^{\alpha}}^{i^{\alpha}} \delta_{i j} & 2{ }^{2} D_{j^{\alpha} i^{\beta}}^{j^{\alpha} \beta^{\beta}} \\
2{ }^{2} D_{i^{\alpha} j^{i^{\alpha}} j^{\beta}} & 2{ }^{2} D_{i^{\beta} j^{\beta}}^{i^{\beta} j^{\beta}}+{ }^{1} D_{i^{\beta}}^{i^{\beta}} \delta_{i j}
\end{array}\right),
\end{aligned}
$$

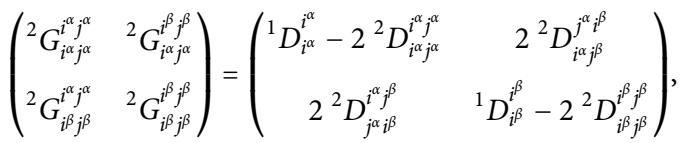

$$
\begin{aligned}
& { }^{2} G_{i^{\sigma} j^{\tilde{\sigma}}}^{i^{\sigma} j^{\tilde{\sigma}}}={ }^{1} D_{i^{\sigma}}^{i^{\sigma}}-2{ }^{2} D_{i^{\sigma} j^{\tilde{\sigma}}}^{i^{\sigma} j^{\tilde{\sigma}}} \quad(\sigma=\alpha, \beta) .
\end{aligned}
$$

Each block of the matrix ${ }^{2} G$ must be positive semidefinite.

The $T 1$ condition is

$$
\begin{aligned}
(T 1)_{j^{\sigma_{2}} l^{\sigma_{4}} n^{\sigma_{6}}}^{i_{\sigma_{5}}^{\sigma_{5}}} & =\left\langle\Psi\left|a_{i^{\sigma_{1}}}^{\dagger} a_{k^{\sigma_{3}}}^{\dagger} a_{m^{\sigma_{5}}}^{\dagger} a_{n^{\sigma_{6}}} a_{l^{\sigma_{4}}} a_{j^{\sigma_{2}}}+a_{j^{\sigma_{2}}} a_{l^{\sigma_{4}}} a_{n^{\sigma_{6}}} a_{m^{\sigma_{5}}}^{\dagger} a_{k^{\sigma_{3}}}^{\dagger} a_{i^{\sigma_{1}}}^{\dagger}\right| \Psi\right\rangle \\
& \geq 0\left(\sigma_{1}, \sigma_{2}, \sigma_{3}, \sigma_{4}, \sigma_{5}, \sigma_{6}=\alpha, \beta\right) .
\end{aligned}
$$

The $T 1$ matrix elements satisfying the spin-coordinate distribution $\left(\sigma_{1}+\sigma_{3}+\sigma_{5} \neq \sigma_{2}+\sigma_{4}+\sigma_{6}\right)$ are zero.

The $T 2$ condition is

$$
\begin{aligned}
(T 2)_{j^{\sigma_{2}} l^{\sigma_{4}} n^{\sigma_{6}}}^{i_{\sigma_{3}}^{\sigma_{3}}} & =\left\langle\Psi\left|a_{i^{\sigma_{1}}}^{\dagger} a_{k^{\sigma_{3}}}^{\dagger} a_{m^{\sigma_{5}}} a_{n^{\sigma_{6}}}^{\dagger} a_{l^{\sigma_{4}}} a_{j^{\sigma_{2}}}+a_{n^{\sigma_{6}}}^{\dagger} a_{l^{\sigma_{4}}} a_{j^{\sigma_{2}}} a_{i^{\sigma_{1}}}^{\dagger} a_{k^{\sigma_{3}}}^{\dagger} a_{m^{\sigma_{5}}}\right| \Psi\right\rangle \\
& \geq 0\left(\sigma_{1}, \sigma_{2}, \sigma_{3}, \sigma_{4}, \sigma_{5}, \sigma_{6}=\alpha, \beta\right) .
\end{aligned}
$$

These T2 matrix elements are zero when the following spincoordinate distribution is satisfied $\left(\sigma_{1}+\sigma_{3}+\sigma_{6} \neq \sigma_{2}+\sigma_{4}\right.$ $\left.+\sigma_{5}\right)^{5}$

These spin restrictions allow one to express the matrices $T 1$ and $T 2$ as a direct sum of submatrices, which must be positive semidefinite. The spin blocks of these matrices, in the DOCI case, have been reported in Ref. 23

Moreover, it has been taken into account that ${ }^{1} \mathrm{D},{ }^{1} \mathrm{Q},{ }^{2} \mathrm{P},{ }^{2} \mathrm{Q},{ }^{2} \mathrm{G}$, $T 1$, and $T 2$ are Hermitian matrices and antisymmetric with respect to all pair and triple creation indices (or annihilation ones) in the case of ${ }^{2} P,{ }^{2} Q$, and $T 1$ matrices. The matrix $T 2$ must be antisymmetric with respect to the first two indices of each trio. The $T 2^{\prime}$ condition, which has been used in Ref. 23 , turns out to be identical to the $T 2$ one not only for the RDOCI scheme but also for the UDOCI one, and consequently, it has not been considered in this work. A more detailed expression for the matrix elements of the conditions $T 1$ and $T 2$ is also indicated in that reference.

Finally, it must be noted that the elements of the spin blocks corresponding to the 2-RDM arising from the UDOCI wave functions satisfy the relationships

$$
2{ }^{2} D_{i^{\alpha} j^{\alpha}}^{i^{\alpha} j^{\alpha}}=2{ }^{2} D_{i^{\beta} j^{\beta}}^{i^{\beta} j^{\beta}}=2{ }^{2} D_{i^{\alpha} j^{\beta}}^{i^{\alpha} j^{\beta}}-\delta_{i}^{j} D_{j^{\alpha}}^{i^{\alpha}}
$$

Consequently, the semidefinite program may be reformulated by considering a lower number of variables, following a similar procedure to that outlined in Ref. 52. In fact, our codes have been programed taking into account those relationships.

\section{REFERENCES}

${ }^{1}$ R. McWeeny, Rev. Mod. Phys. 32, 335 (1960).

${ }^{2}$ A. J. Coleman, Rev. Mod. Phys. 35, 668 (1963).

${ }^{3}$ A. J. Coleman, Int. J. Quantum Chem. 11, 907 (1977).

${ }^{4}$ C. Garrod and J. K. Percus, J. Math. Phys. 5, 1756 (1964).

${ }^{5}$ Z. Zhao, B. J. Braams, M. Fukuda, M. L. Overton, and J. K. Percus, J. Chem. Phys. 120, 2095 (2004).

${ }^{6}$ J. R. Hammond and D. A. Mazziotti, Phys. Rev. A 71, 062503 (2005).

${ }^{7}$ J. R. Hammond and D. A. Mazziotti, Phys. Rev. A 73, 012509 (2006).

${ }^{8}$ D. A. Mazziotti, Phys. Rev. A 74, 032501 (2006).

${ }^{9}$ M. Nakata, B. J. Braams, K. Fujisawa, M. Fukuda, J. K. Percus, M. Yamashita, and Z. Zhao, J. Chem. Phys. 128, 164113 (2008).

${ }^{10}$ F. Weinhold and E. B. Wilson, J. Chem. Phys. 46, 2752 (1967).

${ }^{11}$ F. Weinhold and E. B. Wilson, J. Chem. Phys. 47, 2298 (1967).

${ }^{12}$ P. Ring and P. Schuck, The Nuclear Many-Body Problem (Springer-Verlag, New York, 1980).

${ }^{13}$ D. S. Koltun and J. M. Eisenberg, Quantum Mechanics of Many Degrees of Freedom (Wiley, New York, 1988)

${ }^{14}$ T. Pérez and P. Cassam-Chenaï, J. Math. Chem. 56, 1428 (2018).

${ }^{15}$ L. Bytautas, T. M. Henderson, C. A. Jiménez-Hoyos, J. K. Ellis, and G. E. Scuseria, J. Chem. Phys. 135, 044119 (2011).

${ }^{16}$ D. R. Alcoba, A. Torre, L. Lain, G. E. Massaccesi, and O. B. Oña, J. Chem. Phys. 139, 084103 (2013).

${ }^{17}$ D. R. Alcoba, A. Torre, L. Lain, G. E. Massaccesi, and O. B. Oña, J. Chem. Phys. 140, 234103 (2014).

${ }^{18}$ D. R. Alcoba, A. Torre, L. Lain, O. B. Oña, P. Capuzzi, M. Van Raemdonck, P. Bultinck, and D. Van Neck, J. Chem. Phys. 141, 244118 (2014)

${ }^{19}$ D. R. Alcoba, A. Torre, L. Lain, O. B. Oña, G. E. Massaccesi, and P. Capuzzi, Adv. Quantum Chem. 76, 315 (2018).

${ }^{20}$ L. Bytautas and J. Dukelsky, Comput. Theor. Chem. 1141, 74 (2018).

${ }^{21}$ D. R. Alcoba, A. Torre, L. Lain, G. E. Massaccesi, O. B. Oña, and P. Capuzzi, J. Chem. Phys. 145, 014109 (2016).

${ }^{22}$ W. Poelmans, M. Van Raemdonck, B. Verstichel, S. De Baerdemacker, A. Torre, L. Lain, G. E. Massaccesi, D. R. Alcoba, P. Bultinck, and D. Van Neck, J. Chem. Theory Comput. 11, 4064 (2015).

${ }^{23}$ D. R. Alcoba, A. Torre, L. Lain, G. E. Massaccesi, O. B. Oña, E. M. Honoré, W. Poelmans, D. Van Neck, P. Bultinck, and S. De Baerdemacker, J. Chem. Phys. 148, 024105 (2018).

${ }^{24}$ A. Rubio-García, D. R. Alcoba, P. Capuzzi, and J. Dukelsky, J. Chem. Theory Comput. 14, 4183 (2018).

${ }^{25}$ D. R. Alcoba, P. Capuzzi, A. Rubio-García, J. Dukelsky, G. E. Massaccesi, O. B. Oña, A. Torre, and L. Lain, J. Chem. Phys. 149, 194105 (2018).

${ }^{26}$ M. Yamashita, K. Fujisawa, M. Fukuda, K. Kobayashi, K. Nakata, and M. Nakata, "Latest developments in the SDPA family for solving large-scale SDPs," in Handbook on Semidefinite, Conic and Polynomial Optimization (Springer International Publishing AG, 2012), Vol. 166, pp. 687-713. 
${ }^{27}$ P. A. Limacher, T. D. Kim, P. W. Ayers, P. A. Jonhson, S. De Baerdemacker, D. Van Neck, and P. Bultinck, Mol. Phys. 112, 853 (2014).

${ }^{28}$ M. Van Raemdonck, D. R. Alcoba, W. Poelmans, S. De Baerdemacker, A. Torre, L. Lain, G. E. Massaccesi, D. Van Neck, and P. Bultinck, J. Chem. Phys. 143, 104106 (2015)

${ }^{29}$ L. Lain, A. Torre, D. R. Alcoba, O. B. Oña, and G. E. Massaccesi, Theor. Chem. Acc. 134, 85 (2015).

${ }^{30}$ D. R. Alcoba, A. Torre, L. Lain, G. E. Massaccesi, O. B. Oña, P. W. Ayers, M. Van Raemdonck, P. Bultinck, and D. Van Neck, Theor. Chem. Acc. 135, 153 (2016).

${ }^{31}$ K. Head-Marsden and D. A. Mazziotti, J. Chem. Phys. 147, 084101 (2017).

${ }^{32}$ J. Paldus, P. Piecuch, L. Pylypow, and B. Jeziorski, Phys. Rev. A 47, 2738 (1993).

${ }^{33}$ T. Van Voorhis and M. Head-Gordon, J. Chem. Phys. 113, 8873 (2000).

${ }^{34}$ M. Nakata, M. Ehara, and H. Nakatsuji, J. Chem. Phys. 116, 5432 (2002).

${ }^{35}$ C. Valdemoro, D. R. Alcoba, O. B. Oña, L. M. Tel, E. Perez-Romero, and J. M. Oliva, Chem. Phys. 399, 59 (2012).

${ }^{36}$ J. K. Ellis, R. L. Martin, and G. E. Scuseria, J. Chem. Theory Comput. 9, 2857 (2013).

${ }^{37}$ E. Ramos-Cordoba, X. Lopez, M. Piris, and E. Matito, J. Chem. Phys. 143, 164112 (2015).

${ }^{38}$ P. R. Surjan, Second Quantized Approach to Quantum Chemistry, An Elementary Introduction (Springer-Verlag, Berlin, 1989).
${ }^{39}$ P. Karafiloglou, J. Chem. Phys. 130, 164103 (2009)

${ }^{40}$ D. R. Alcoba, R. C. Bochicchio, L. Lain, and A. Torre, Chem. Phys. Lett. 429 286 (2006).

${ }^{41}$ T. M. Henderson and G. E. Scuseria, Phys. Rev, A 96, 022506 (2017).

${ }^{42}$ C. Valdemoro, L. M. Tel, D. R. Alcoba, E. Perez-Romero, and F. J. Casquero, Int. J. Quantum Chem. 90, 1555 (2002).

${ }^{43}$ D. R. Alcoba and C. Valdemoro, Int. J. Quantum Chem. 102, 629 (2005).

${ }^{44}$ D. R. Alcoba, in Two-Electron Reduced-Density-Matrix Mechanics: With Application to Many-Electron Atoms and Molecules, Advances in Chemical Physics, Vol. 134, edited by D. A. Mazziotti (Wiley, New York, 2007), Chap. 10.

${ }^{45}$ J. F. Stanton, J. Chem. Phys. 101, 371 (1994).

${ }^{46}$ A. I. Krylov, J. Chem. Phys. 113, 6052 (2000).

${ }^{47}$ T. D. Crawford, C. D. Sherrill, E. F. Valeev, J. T. Fermann, R. A. King, M. L. Leininger, S. T. Brown, C. L. Janssen, E. T. Seidl, J. P. Kenny, and W. D. Allen, J. Comput. Chem. 28, 1610 (2007).

${ }^{48}$ G. Knizia and G. K.-L. Chan, Phys. Rev. Lett. 109, 186404 (2012).

${ }^{49}$ G. Knizia and G. K.-L. Chan, J. Chem. Theory Comput. 9, 1428 (2013).

${ }^{50}$ A. Margócsy, P. Kowalski, K. Pernal, and A. Szabados, Theor. Chem. Acc. 137, 159 (2018).

${ }^{51} \mathrm{Z}$. Zhao, "The reduced density matrix method for the electronic structure calculations: Applications of semidefinite programming to N-fermion systems," Ph.D. thesis, New York University, 2004.

${ }^{52}$ P. A. Limacher, J. Chem. Theory Comput. 11, 3629 (2015). 